\title{
Effect of Remote Glucose Monitoring Utilizing Computerized Insulin Dose Adjustment Algorithms: A Pilot Project
}

\author{
Mayer B. Davidson · S. Joshua Davidson
}

Received: October 4, 2018 / Published online: February 5, 2019

(C) The Author(s) 2019

\begin{abstract}
Introduction: Primary care physicians are often challenged to adjust insulin doses. To facilitate this process, we evaluated in a safety net clinic the work flow and glycemic effects of remote glucose monitoring utilizing Federal Drug Administration (FDA) cleared, Conformité Européenne (CE) registered software that contained computerized algorithms for insulin dose adjustments to help clinicians make dosing decisions for insulin-requiring patients.

Methods: Patients taking insulin for at least 6 months with $\mathrm{HbA}_{\mathrm{c}}$ levels of at least $8.0 \%$ measured glucose levels with a meter attached to their smartphones. Readings were automatically transmitted to a secure, Health Insurance Portability and Accountability Act (HIPAA)-approved server. Values were analyzed every $2-3$ weeks and reports, including recommendations for insulin
\end{abstract}

Enhanced Digital Features To view enhanced digital features for this article go to https://doi.org/10.6084/ m9.figshare.7547411.

Electronic supplementary material The online version of this article (https://doi.org/10.1007/s13300019-0565-y) contains supplementary material, which is available to authorized users.

M. B. Davidson ( $\square)$

Charles R. Drew University, Los Angeles, CA, USA

e-mail: mayerdavidson@cdrewu.edu

M. B. Davidson · S. J. Davidson

Mellitus Health Inc., Los Angeles, CA, USA dose changes, were sent to a clinic nurse practitioner (NP) who modified or accepted the recommendations. A staff person contacted patients with the new doses determined by the NP.

Results: Insulin regimens included basal alone $(N=11)$, basal/bolus $(N=14)$, and self-mixed/ split $(N=3)$. Baseline HbA1 levels of $10.0 \%$ fell to $8.1 \%$ at 3 months $(N=28)$ and $7.6 \%$ at 6 months $(N=17)$ without any clinic visits for dose adjustments. There were 268 reports which allowed providers to see 268 other patients during these avoided clinic visits. The NP agreed with $82 \%$ of the recommendations. The total doses of insulin increased by $24 \%$. No patient experienced severe hypoglycemia or visited an emergency department for hypoglycemia.

Conclusion: Remote glucose monitoring utilizing computerized insulin dose adjustment algorithms saved time for both providers and patients while effectively improving glycemia.

Funding: The Leonard M. Lipman Charitable Trust and Mellitus Health.

Keywords: Computerized insulin dose adjustment algorithms; Remote glucose monitoring; Telemedicine

\section{INTRODUCTION}

Insulin secretion is reduced on average by $50 \%$ when type 2 diabetes is diagnosed [1] and progressively decreases despite ongoing anti- 
hyperglycemia therapy [2]. This scenario results in easy control of diabetes initially, usually with just one non-insulin drug, but subsequent requirements for 2-4 additional ones. Eventually, insulin secretion often decreases to the point where non-insulin drugs are unable to control type 2 diabetic patients and insulin is necessary. Currently, approximately $30 \%$ of Caucasians and African Americans and 22\% of Latinos are receiving insulin [3]. Ninety percent of diabetic patients are cared for by primary care physicians [4] who are reluctant to start insulin, and even if initiated, it is often either discontinued or fails to be intensified [5-12]. Physicians state that a major factor in their reluctance to start and subsequently intensify insulin treatment is due to time constraints $[5,7,13,14]$. Because of the number of patients that they are following, time constraints also involve less frequent visits with most patients being routinely seen every 3 months or so. This is particularly problematic for insulin-requiring diabetic patients. Two-thirds of patients on insulin fail to achieve the American Diabetes Association's (ADA) target $\mathrm{HbA} 1_{\mathrm{c}}$ level of below $7.0 \%$ [15]. Yet a clinical trial showed that if insulin doses were adjusted every 1-4 weeks, $88 \%$ of patients reached that goal [15].

Remote glucose monitoring in which glucose readings are transmitted to the patient's provider in a format that facilitates insulin dose adjustments has great potential of saving time for both providers and patients as well as increasing the frequency of interactions between the two. We utilized a system in which a glucose meter attached to a smartphone containing a program downloaded from the Web that automatically sent every glucose reading to a secure, Health Insurance Portability and Accountability Act (HIPAA)-approved cloud server. We carried out a work flow project with this tool in a safety net, community clinic whose population is $57 \%$ Hispanic or Latino, $14 \%$ homeless, and $76 \%$ below the federal poverty level. In this clinic, $24 \%$ of diabetic patients have $\mathrm{HbA} 1_{c}$ levels exceeding 9.0\%, the vast majority of whom are receiving insulin. These patients are usually seen every 3 months.

The aim of the work flow project was to evaluate the amount of time saved by utilizing remote glucose monitoring compared to faceto-face visits for insulin-requiring patients. $\mathrm{HbA} 1_{\mathrm{c}}$ levels were measured to help the clinic decide whether to adopt this approach should the work flow changes seem beneficial.

\section{METHODS}

Patients eligible for the study were $18-75$ years of age, had been taking insulin for at least 6 months with an $\mathrm{HbA} 1_{\mathrm{c}}$ level within the past month of at least $8.0 \%$, and used a smartphone. They were given an iHealth Align glucose meter with an associated mobile application that attaches to a smartphone. The mobile application automatically transmitted each glucose reading to the account of the user in the iHealth cloud system. Mellitus Health's server was notified each time that there was a new reading for that patient. Two to 3 weeks after the last contact with the patient by the staff person (which is noted by the Mellitus Health server), the server requested the glucose values (along with the date and time that they were measured) from the iHealth server that had been obtained since the last report was generated. These glucose readings were then presented as a scattergram in addition to being listed as preand post-prandial and before bedtime values and sent to a designated staff person in the clinic. This report, received electronically by the staff person, was printed and shown to an assigned nurse practitioner (NP) who decided if any insulin dose adjustments were necessary and had the staff person contact the patient with either any new doses or maintenance of the previous ones.

After enrollment, patients could be discharged from the remote glucose monitoring project for one of three reasons: (1) if they were found to consistently not take the recommended insulin doses, i.e., the staff person determined on calling the patient after three separate reports that the exact recommended insulin doses were not being taken; (2) if they could not be contacted over 9 weeks despite many attempts to reach them; and (3) if there were too few readings [16] over 6 weeks during all four periods (morning, afternoon, evening, 
overnight) that are maximally covered by the various insulin preparations in the patient's regimen. For instance, in patients on basal/bolus, self-mixed/split, or pre-mixed insulin regimens, each period is maximally affected by a separate component of the insulin regimen. For a patient to be discharged, there would need to be too few readings in every one of the periods over 6 weeks. Conversely, patients would remain in the study if any one period contained enough readings for analysis regardless of the insulin regimen.

After the NP reviewed the report, she either accepted or modified the recommendations and returned it to the staff person. The staff person then called the patient to inform them of the new insulin doses after ascertaining that indeed they were taking the doses stated in the report for the previous 2-3 weeks. The staff person inquired about any suspiciously high or low glucose values identified by NP that did not seem to fit into the general pattern. Occasionally, the NP changed her decision when that information was made known to her. Finally, the staff person entered the new insulin doses into the electronic health record.

The staff person had only an initial face-toface visit with patients to give them the meter and strips and to instruct them how they should be used in conjunction with the smartphones. All other subsequent contact occurred via phone. No specific modification of lifestyle was suggested. Patients on basal insulin alone were asked to test at least before breakfast each day and those taking two or more injections, at least twice a day before meals and their bedtime snack. (All insulin-requiring patients at this safety net, community clinic are instructed to ingest a small bedtime snack to minimize overnight hypoglycemia.)

The senior author has been teaching medical students and house officers how to adjust insulin doses for over 50 years and registered nurses, NPs, physician assistants, and clinical pharmacists for approximately 35 years. These principles [17], acknowledged by the ADA to meet their standards of care [16], were recently computerized. Only after all of the patients for the work flow project had been enrolled did these computerized dose adjustment algorithms receive Federal Drug Administration (FDA) clearance [18] as well as Conformité Européenne (CE) registration [19]. In addition to the scattergram and organized glucose readings described above, the NP could then utilize the full report (see Supplementary Information) which also contained an analysis of the glucose values and suggested recommendations for adjusting insulin doses (if necessary) that she could modify or accept. As the initial enrollment was for a work flow project, the FDA cleared the algorithms for clinical use, and the de-identified $\mathrm{HbA}_{\mathrm{c}}$ levels are from observational data, institutional review board approval and registration as a clinical trial were not warranted.

Non-insulin drugs were not changed throughout the project. Hypoglycemia was defined by a glucose reading below $70 \mathrm{mg} / \mathrm{dl}$ and severe hypoglycemia as an episode outside of a hospital in which another person was necessary for treatment or a visit to a hospital emergency department (these safety net clinic patients do not utilize urgent care centers).

\section{Statistical Analysis}

After the 6-month work flow project was completed, de-identified $\mathrm{HbA} 1_{\mathrm{c}}$ levels collected within 1 month of enrollment and 3 and 6 months later were evaluated by a one-way repeated analysis of variance based on the $F$ test for time differences across subjects. The data were analyzed using NCSS 12 Statistical Software (2018). NCSS, LLC. Kaysville, Utah, http:// ncss.com/software/ncss. Significant differences from baseline values were accepted as a $P$ value of less than 0.05 (2-tailed). The number of patients who achieved a $\mathrm{HbA} 1_{\mathrm{c}}$ level below $7.5 \%$ was also tallied.

\section{Compliance with Ethics Guidelines}

All insulin dose adjustments carried out in this pilot project in insulin-requiring patients were in accordance with Good Clinical Practices (GCP) and with the 1964 Helsinki declaration and its later amendments or comparable ethical standards. 


\section{RESULTS}

The flow of eligible patients, enrolled patients, and completed patients is shown in Fig. 1. A large number of eligible patients were not interested or could not be contacted (it is often difficult to contact patients in this safety net clinic because many travel to Mexico for extended periods and a number are undocumented). Of those eligible for enrollment, lack of a smartphone or inability to use the combined smartphone/iHealth Align glucose meter

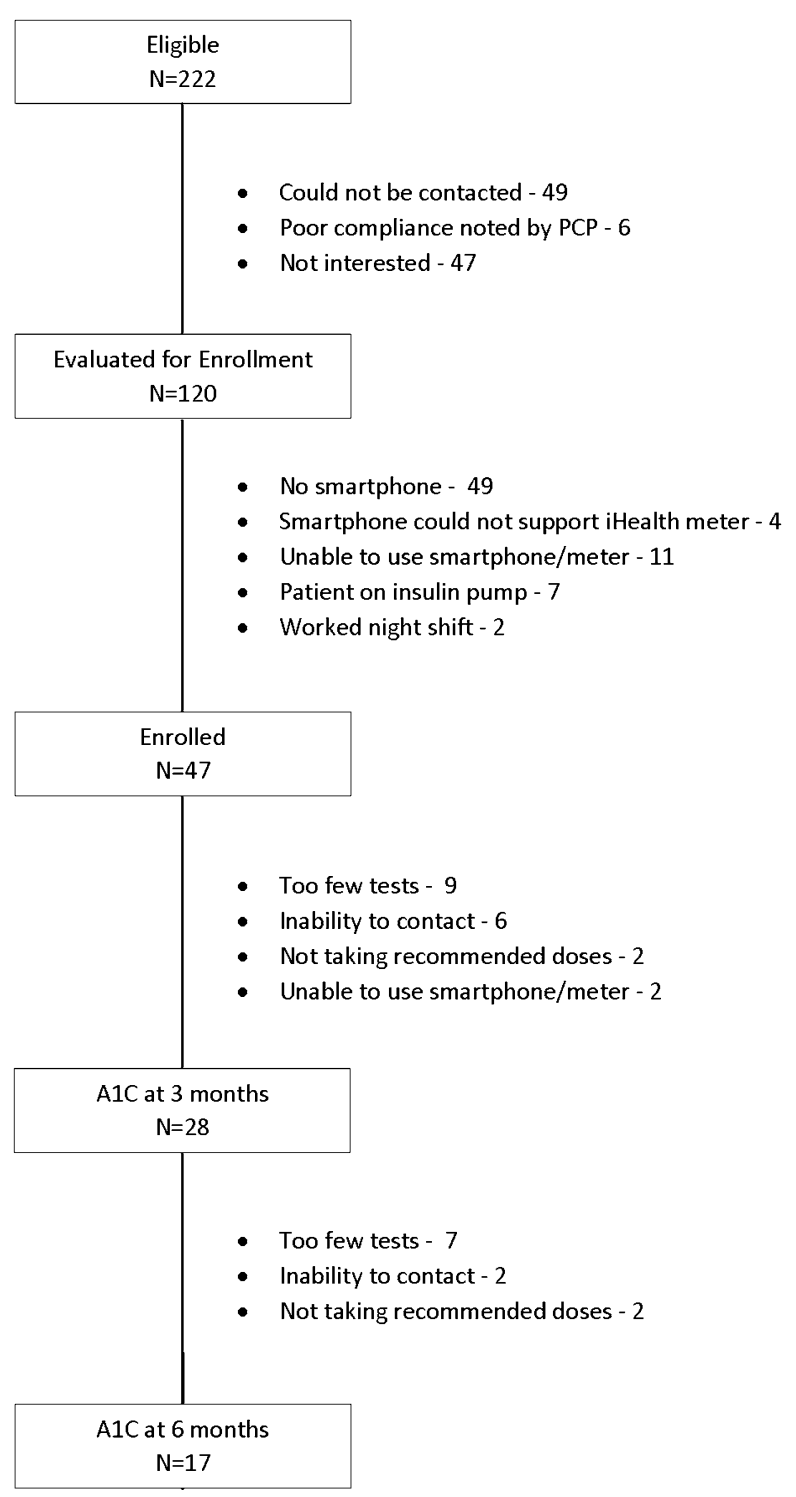

Fig. 1 Diagram of patient selection and flow eliminated another large number of potential participants. After enrollment, the main reasons for dismissal between baseline and 3 months and between 3 and 6 months were mostly too few tests, followed by inability to contact the patient.

The 17 patients followed for 6 months and the 11 patients followed for only 3 months generated 268 reports. The NP spent about 3 min reviewing each report for a total of $804 \mathrm{~min}$ or $13.4 \mathrm{~h}$ over the 6-month project. Patient visits at the clinic are slotted for $15 \mathrm{~min}$ each. If these patients had been seen, these 268 visits would have consumed $67 \mathrm{~h}$. Thus, remote glucose monitoring with the reports sent to the NP allowed the patients' providers 268 clinic visits for other patients at a cost of $13.4 \mathrm{~h}$ of the NP's time.

The staff person spent about $45 \mathrm{~min}$ at the initial (and only face-to-face) visit explaining the project, educating the patient on how to use the combined smartphone/glucose meter, detailing the requirements of glucose testing, and describing the process of contacting the patient. About 15-20 min were spent on explaining how to use the combined smartphone/meter and the testing requirements. She spent 3-5 min on each phone contact with the patient and about 10 min transferring the NPs new dose recommendations into the electronic health record as well as updating the insulin doses in the medication section. After the initial visit, this came to a total of $1072 \mathrm{~min}$ or $17.9 \mathrm{~h}$ for these 28 patients (using $4 \mathrm{~min}$ per interaction for this calculation). Of course, this does not include the time spent on unsuccessful attempts to reach patients.

The average time for a patient visit at the Venice Family Clinic from registration to leaving the clinic is $101 \mathrm{~min}$ or $1.7 \mathrm{~h}$ (this includes picking up medication from the pharmacy). By not having to come for face-to-face visits at the frequency of the generated reports, these 28 patients saved $456 \mathrm{~h}$. This does not include the transportation time or waiting to be registered as well as any costs (transportation or child care) or inconveniences to family members or friends.

The demographics of the participants are shown in Table 1. Twenty-seven patients had 
Table 1 Demographics

\begin{tabular}{ll}
\hline Age (years \pm SD) & $55.9 \pm 8.6$ \\
Race/ethnicity & 20 \\
Hispanic & 4 \\
Non-Hispanic Black & 3 \\
White & 1 \\
Asian & \\
Sex & 15 \\
Female & 13 \\
Male & \\
Insulin regimen & 11 \\
Basal alone & 14 \\
Basal/bolus & 3 \\
Self-mixed/split &
\end{tabular}

28 patients with 3 -month $\mathrm{HbAl}_{\mathrm{c}}$ measurements

type 2 diabetes and 1 patient had type 1 diabetes. Their baseline $\mathrm{HbA1}_{\mathrm{c}}( \pm \mathrm{SD})$ levels of $10.0 \% \pm 1.2$ fell $1.9 \%$ to $8.1 \% \pm 1.0$ at 3 months and another $0.5 \%$ to $7.6 \% \pm 0.8$ at 6 months, $P<10^{-6}$ (Fig. 2). The decrease in $\mathrm{HbA}_{\mathrm{c}}$ levels were comparable among all 3 insulin regimens. Baseline and final values in the 11 patients on basal insulin alone were

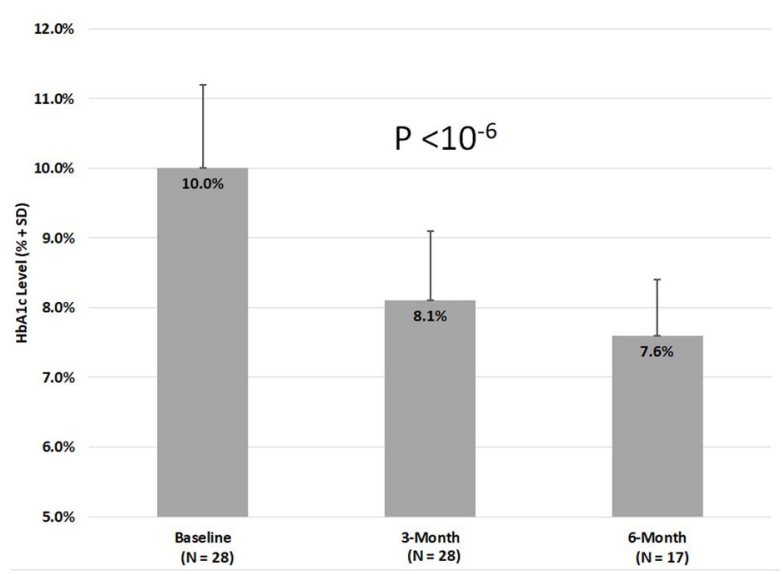

Fig. $2 \mathrm{HbAl}_{\mathrm{c}}$ responses to remote glucose monitoring utilizing computerized insulin dose adjustment algorithms
$10.0 \%$ and $8.2 \%$, in the 14 patients on basal/ bolus insulin were $9.8 \%$ and $7.8 \%$ and the 3 patients on self-mixed/split insulin were $10.9 \%$ and $8.0 \%$, respectively. After the initial education visit to learn how to use the system, there were no clinic visits for adjustment of insulin doses. Thirty-six percent of the patients achieved a $\mathrm{HbA}_{\mathrm{c}}$ level below $7.5 \%$ and $50 \%$ achieved below $8.0 \%$.

There were 765 recommendations in 268 generated reports. The NP accepted 590 and modified 175 of them. Twenty-six of the "modifications" occurred when the staff person ascertained that the patient was not taking insulin as recommended, 19 when the patient could not be contacted so that any dose adjustment recommendations could not take place, and 2 when the staff person determined that the patients' meal times were not recorded accurately. This left 718 recommendations with which the NP agreed in 590 (82\%) and disagreed in $128(18 \%)$. The reasons for the disagreements are listed in Table 2. The most common reason for disagreement was that the NP thought there were enough readings to make an adjustment

Table 2 Reasons for disagreements between the algorithms (A) and the nurse practitioner (NP) in 718 actionable recommendations

\begin{tabular}{ll}
\hline Reason & Number \\
\hline NP thought enough readings for change; A did & 66 \\
not & \\
A thought enough readings for change; NP did & 18 \\
not & 16 \\
NP weighted low readings more than A & 9 \\
More recent readings at target: NP no change; & 9 \\
A increased based on all readings & 8 \\
NP changed A dose by 1 unit to achieve even & 8 \\
dose for syringe users & 6 \\
NP did not allow SARA in patients on basal & 6 \\
alone while A recommended it & 5 \\
NP increased dose less than A
\end{tabular}

$S A R A$ short- or rapid-acting insulin 
while the computerized algorithms did not. This reflects a more conservative approach by the algorithms than the NP. On the basis of the NP's changes in insulin doses, the total amount of insulin used by these 28 patients increased by $24 \%$ by the end of the remote glucose monitoring project.

These 28 patients recorded 4671 blood glucose readings averaging only 1.2 tests per day. Regarding hypoglycemia, 415 or $9 \%$ of them were below $70 \mathrm{mg} / \mathrm{dl}$. Twenty-six of the patients had at least one episode of hypoglycemia. Averaged over all patients, this translates into 35 episodes per year per patient. There were no episodes of severe hypoglycemia nor visits to an emergency department for hypoglycemia. Further, as is usually the case, a small number of patients were responsible for the majority of the hypoglycemic episodes; two patients accounted for $30 \%$ and five patients accounted for $52 \%$ of the hypoglycemic readings.

Baseline and final weights were available for 26 patients. These patients gained only $0.7 \mathrm{~kg}$ $(82.0$ vs $82.7 \mathrm{~kg})$.

\section{DISCUSSION}

The large amount of time saved and the convenience for patients who use remote glucose monitoring are obvious. The advantages for providers are threefold. First, it frees up time to see other patients. Second, it increases the frequency of interactions with insulin-requiring patients. Third, the organization of the glucose readings into pre- and post-prandial and before bedtime values (and possibly the scattergram) facilitates decision-making for the provider. For those who utilize the algorithms, the analysis of the values and the recommendations that can be modified or accepted further facilitate the provider's ability to adjust insulin doses. With the exception of the first advantage, all of the rest should improve glycemia in insulin-requiring patients.

Remote glucose monitoring utilizing computerized insulin dose adjustment algorithms in these insulin-requiring patients lowered $\mathrm{HbA} 1_{\mathrm{c}}$ levels from $10.0 \%$ to $8.1 \%$ after 3 months and to $7.6 \%$ after 6 months (Fig. 2) in this mostly minority, safety net clinic population. An earlier version of these computerized insulin dose adjustment algorithms was used by a registered nurse trained on them who lowered $\mathrm{HbA} 1_{\mathrm{c}}$ levels from $8.4 \%$ to $7.7 \%$ over 20 weeks in 29 remotely monitored, insulin-requiring patients, 10 of whom had type 1 diabetes [20]. Another registered nurse trained to use these dosing algorithms (non-computerized) and supervised by primary care physicians in a family medicine clinic serving a similar safety net population decreased baseline $\mathrm{HbA} 1_{\mathrm{c}}$ levels in 111 insulinrequiring patients referred to her by these physicians from $11.0 \%$ to $7.2 \%$ in $9-12$ months of clinic visits [21].

The vast majority of currently available insulin dosing recommendations calculate preprandial (bolus) doses based on the estimated carbohydrate (carb) content of the meal and the prevailing glucose concentrations. Besides being difficult to learn, taking up to 6 months to allegedly becoming proficient, carb counting by patients is not that accurate. Compared to a registered dietitian, patients are off by $20-40 \%$ $[22,23]$. Furthermore, recent studies have shown that the protein and lipid content of a meal could increase a bolus insulin dose based on just the carb content of the meal by up to $65 \%[24,25]$. A systemic assessment of 46 smartphone apps for calculating bolus doses concluded that the majority of them provided no protection against and may actively have contributed to incorrect or inappropriate dose recommendations [26].

The use of these clinician-involved computerized insulin dose adjustment algorithms follows a different approach. Although stress on improving dietary and exercise lifestyles should continue, the dosing recommendations are based on analyzing the pattern of glucose values obtained during patients' current lifestyles. If the lifestyle should improve (or worsen), the glucose patterns would change accordingly and the appropriate changes in insulin doses would be recommended. These computerized insulin dose adjustment algorithms incorporate all approved insulin preparations and cover eight different standard insulin regimens: basal insulin alone, bedtime NPH insulin alone, basal/ bolus, self-mixed/split, pre-mixed, U-500 
regular, and the unusual delayed response to $\mathrm{NPH}[27,28]$ and U-500 regular [29] insulins. Additionally, they recommend intensification of insulin when appropriate. For instance, if the glucose values during a particular period are high and there is no insulin preparation in the regimen to cover that period, the recommendations will suggest adding an injection of an appropriate insulin type that will lower those elevated glucose readings.

A crucial reason for the effectiveness of this remote glucose monitoring pilot project is the increased interactions between the patient and clinician so that more frequent insulin dose adjustments can be made. In contrast to only one-third of insulin-requiring patients achieving the $\mathrm{ADA} \mathrm{HbA} 1_{\mathrm{c}}$ target of below $7.0 \%$ under usual care, $88 \%$ reached that target when insulin dose adjustments were made every 1-4 weeks [15]. In addition to increased effectiveness, frequent evaluations for insulin dose changes are necessary to avoid hypoglycemia. Over the course of a year, two-thirds of patients required a more than $20 \%$ decrease in their insulin requirements [30]. The period of decreased insulin doses lasted 10 weeks and the total insulin dose was decreased by $41 \%$. The usual frequency of insulin dose adjustments of 2-4 times per year places insulin-requiring patients at an increased risk for hypoglycemia.

Our rate of hypoglycemia was similar to others who intensify insulin treatment. In the AUTONOMY study [31] in which a basal/bolus regimen was initiated in patients who had failed basal insulin alone, $86-98 \%$ of patients experienced at least one episode of hypoglycemia. The yearly rate of hypoglycemic episodes in that study ranged from 38 to 51 per patient per year, compared to our rate of 35 . In contrast to the weight gain seen soon after insulin therapy is initiated, our $0.7-\mathrm{kg}$ increase in weight is consistent with the minimal weight gain occurring after the first year of insulin therapy [32].

If remote glucose monitoring is not feasible, the computerized insulin dose adjustment algorithms can be used at office visits. A program is placed on an office computer, or if possible, into the electronic health record. The glucose meter is downloaded into that computer and within $15 \mathrm{~s}$ the same report is generated. An additional feature of this approach is that a "day view" of the glucose readings is available to the clinician who can delete outlier values (after talking with the patient about the reason for them) before the analysis. Although these interactions would usually be less frequent than the remote monitoring ones, and therefore would take longer to bring $\mathrm{HbA}_{\mathrm{c}}$ levels to target, there would be large savings of time, especially if front office staff downloaded the glucose meter and presented the report to the clinician. The use of these computerized insulin dose adjustment algorithms at office visits would give the clinician more time to focus on other aspects of patient care in the limited time available.

Although the principles underlying these insulin dose adjustments have been used successfully for many years by mid-level providers, their computerization requires documentation of their safety, i.e., no increased risk of hypoglycemia, as well as their effectiveness. The results of this pilot project attest to their effectiveness. The algorithms limit the amount that each insulin dose can be increased [16] which protects against excessive hypoglycemia. The slightly decreased number of episodes of hypoglycemia in this pilot project compared to the AUTONOMY study that also intensified insulin treatment attests to their safety. Of course, further studies will be necessary for confirmation.

A limitation of this project is that it will be difficult to extrapolate these results to the general population since it was carried out in a safety net clinic whose challenging population can be difficult to contact and is often reluctant to perform ongoing glucose testing. Many of them did not have smartphones, and of those who did, a number found it difficult to use the combined smartphone and attached glucose meter. Of the 120 patients eligible for enrollment, over half could not be enrolled because of smartphone issues. Of the 47 who were enrolled, only 17 successfully completed the 6-month pilot project (Fig. 1). On the other hand, this pilot project demonstrated that if patients were knowledgeable in using their smartphones, tested routinely, could be contacted, and followed recommended insulin dose changes, $\mathrm{HbA} 1_{\mathrm{c}}$ levels could be markedly 
lowered in poorly controlled patients. It should be noted that lifestyle education was not emphasized so that any $\mathrm{HbA} 1_{\mathrm{c}}$ changes could be more likely ascribed to this telehealth approach. Any lifestyle improvements would be reflected in subsequent glucose readings and taken into account by the algorithms. Finally, since it was an observational project without a control group, the improved glycemia cannot necessarily be due only to the use of the computerized algorithms. More frequent interactions could have played a role.

There is a compelling need to overcome the clinical inertia that impedes appropriately initiating and intensifying insulin. The reluctance to initiate insulin therapy leads to long delays of up to 7.7 years after patients fail combinations of non-insulin drugs before insulin was started. Even then, many patients did not receive insulin [12, 33-37]. When insulin was started in type 2 diabetic patients, $\mathrm{HbA} 1_{\mathrm{c}}$ levels ranged from $8.9 \%$ through $9.8 \%$, averaging $9.3 \%$ $[33,34,37-40]$, and was $9.7 \%$ when the insulin regimen was intensified $[12,36]$. The $H b A 1_{c}$ levels of patients receiving insulin ranged from $7.9 \%$ through 9.4\%, averaging $8.5 \%$ $[37,38,41,42]$. Even though only two patients achieved the ADA $\mathrm{HbA}_{\mathrm{c}}$ goal of below $7.0 \%$ (data not shown), the mean level of those successfully completing the 6-month pilot project was $0.9 \%$ less than the average value in the less challenged general population of insulin-requiring diabetic patients. A telehealth approach is very likely to be effective in dealing with issues involving insulin therapy.

The direct medical cost of diabetes care in the USA in 2017 was 237 billion dollars [43], a sizeable proportion of which was related to the microvascular complications of diabetes. Since achieving, or nearly reaching, the ADA $\mathrm{HbA} 1_{\mathrm{c}}$ target of below $7.0 \%$ prevented the development or markedly slowed the progression of these microvascular complications [44], use of effective computerized insulin dose adjustment algorithms should decrease the morbidity and mortality related to these complications in insulin-requiring diabetic patients. Furthermore, this improvement in glycemia will save the medical care system large sums. For instance, a $1.0 \%$ drop in $\mathrm{HbA}_{\mathrm{c}}$ levels is associated with a reduction of at least approximately US $\$ 1300$ per patient per year in medical care costs. These savings noted in 1997 [45] were adjusted by the yearly consumer price index (CPI) to 2014. Since the rate of increase of medical care costs is more than the CPI, the yearly savings are likely higher. Thus, the use of computerized insulin dose adjustment algorithms would have both clinical and financial benefits.

\section{CONCLUSION}

Remote glucose monitoring with subsequent analysis of the glucose readings by computerized insulin dose adjustment algorithms generating reports sent to providers containing recommendations for insulin dose changes that can be modified or accepted (1) avoids face-to-face office visits for patients, (2) largely decreases the time spent by providers in adjusting insulin doses, (3) frees up providers to see other patients in the time slots usually reserved for insulin-requiring patients, and (4) markedly lowers $\mathrm{HbA} 1_{\mathrm{c}}$ levels in poorly controlled patients. This kind of telehealth approach should be very effective in meeting the challenges faced by primary care physicians in adjusting insulin doses.

\section{ACKNOWLEDGEMENTS}

The authors gratefully acknowledge Ligaya Scarlett, NP and Jessica M. Goldberg, MPH for their critical role in carrying out this pilot project, the patients who were enrolled in it, and Martin L. Lee for the statistical analysis.

Funding. The personnel for this project were funded by the Leonard M. Lipman Charitable Trust and the article processing charges and the iHealth meter and strips by Mellitus Health, Inc. All authors had full access to all of the data in this study and take complete responsibility for the integrity of the data and accuracy of the data analysis.

Authorship. All named authors meet the International Committee of Medical Journal 
Editors (ICMJE) criteria for authorship for this article, take responsibility for the integrity of the work as a whole, and have given their approval for this version to be published.

Disclosures. Mayer B. Davidson is the Chief Medical Officer of Mellitus Health Inc. S. J. Davidson is the Chief Executive Officer of Mellitus Health, Inc.

Compliance with Ethics Guidelines. All insulin dose adjustments carried out in this pilot project in insulin-requiring patients were in accordance with Good Clinical Practices (GCP) and with the 1964 Helsinki declaration and its later amendments or comparable ethical standards.

Data Availability. The reports based on the remotely generated glucose readings during this pilot project are available from the corresponding author on reasonable request.

Open Access. This article is distributed under the terms of the Creative Commons Attribution-NonCommercial 4.0 International License (http://creativecommons.org/licenses/ by-nc/4.0/), which permits any noncommercial use, distribution, and reproduction in any medium, provided you give appropriate credit to the original author(s) and the source, provide a link to the Creative Commons license, and indicate if changes were made.

\section{REFERENCES}

1. U.K. Prospective Diabetes Study Group. Overview of 6 years' therapy of type II diabetes: a progressive disease. Diabetes. 1995;44:1249-58.

2. Holman RR. Assessing the potential for $\alpha$-glucosidase inhibitors in prediabetic states. Diabetes Res Clin Pract. 1998;40(Suppl):S21-5.

3. Selvin E, Porrinello CM, et al. Trends in insulin use and diabetes control in the U.S.: 1988-1994 and 1999-2012. Diabetes Care. 2016;39:e33-5.

4. Davidson JA. The increasing role of primary physicians in caring for patients with type 2 diabetes mellitus. Mayo Clin Proc. 2010;85(Suppl 12):S3-4.
5. Jeavons D, Hungin APS, Cornford CS. Patients with poorly controlled diabetes in primary care: healthcare clinicians' beliefs and attitudes. Postgrad Med J. 2006;82:347-50.

6. Meece J. Dispelling myths and removing barriers about insulin in type 2 diabetes. Diabetes Educ. 2006;32:1342-9.

7. Cuddihy RM, Philis-Tsimikas A, Nazeri A. Type 2 diabetes care and insulin intensification: is a more multidisciplinary approach needed? Results from the MODIFY survey. Diabetes Educ. 2011;37:111-23.

8. Patrick AR, Fischer MA, Choudry NK, et al. Trends in insulin initiation and treatment intensification among patients with type 2 diabetes. J Gen Intern Med. 2013;29:320-7.

9. Khunti K, Nikolajsen A, Thorsted BL, Andersen M, Davies MJ, Paul SK. Clinical inertia with regard to intensifying therapy in people with type 2 diabetes treated with basal insulin. Diabetes Obes Metab. 2016;18:401-9.

10. Ascher-Svanum H, Lage M, et al. Early discontinuation and restart of insulin in the treatment of type 2 diabetes mellitus. Diabetes Ther. 2014;5:225-42.

11. Bonafede $\mathrm{M}$, Chandran A, DiMano S, et al. Medication usage, treatment intensification, and medical cost in patients with type 2 diabetes: a retrospective database study. BMJ Open Diabetes Res Care. 2016;4:e000189. https://doi.org/10.1136/ bmjdrc-2015-00189.

12. Mata-Cases M, Franch-Nadal J, Real J, et al. Therapeutic inertia in patients treated with two or more antidiabetics in primary care: factors predicting intensification of treatment. Diabetes Obes Metab. 2018;20:103-12.

13. LeBlanc ES, Rosales AG, Kachroo S, et al. Provider beliefs about diabetes treatment have little impact on glycemic control of their patients with diabetes. BMJ Open Diabetes Res Care. 2015;3:e000062. https://doi.org/10.1136/bmjdrc-2014-00062.

14. Ellis K, Mulnier H, Forbes A. Perceptions of insulin use in type 2 diabetes in primary care: a thematic synthesis. BMC Fam Pract. 2018;19(1):70. https:// doi.org/10.1186/s12875-018-0753-2.

15. Bashan E, Herman WH, Hodish I. Are glucose readings sufficient to adjust insulin dosage? Diabetes Tech Therap. 2011;13:85-92.

16. Davidson MB, Hsia S. Glycemia. In: Meeting the American Diabetes Association standards of care: an algorithmic approach to clinical care of the diabetes 
patient. American Diabetes Association, Alexandria, VA, 2017, pp. 76-91.

17. Davidson MB. Insulin therapy: a personal approach. Clin Diabetes. 2015;33:123-35.

18. Food and Drug Administration (FDA). Regulation Number 21 CFR 868 1890: Submission \# k160673.

19. Medicines \& Healthcare Products Regulatory Agency (MHRA), Reference CA015516.

20. Davidson MB, Lewis G. Effect of data management on a central server on $\mathrm{HbA}_{1 \mathrm{c}}$ levels in insulin-requiring patients. Diabetes Care. 2000;23:706-7.

21. Davidson MB, Blanco-Castellanos $M$, Duran $P$. Integrating nurse-directed diabetes management into a primary care setting. Am J Manag Care. 2010;16:652-6.

22. Brazeau AS, Mircescu H, Leroux C, et al. Carbohydrate counting accuracy and blood glucose variability in adults with type 1 diabetes. Diabetes Res Clin Pract. 2013;99:19-23.

23. Meade LT, Rushton WE. Accuracy of carbohydrate counting in adults. Clin Diabetes. 2016;34:142-7.

24. Bell KJ, Toschi E, Steil GM, et al. Optimized mealtime insulin dosing for fat and protein in type 1 diabetes: application of a model-based approach to derive insulin doses for open-loop diabetes management. Diabetes Care. 2016;39:1631-4.

25. Paterson MA, Smart CEM, Lopez PE, et al. Increasing the protein quantity in a meal results in dosedependent effects on postprandial glucose levels in individuals with type 1 diabetes mellitus. Diabetic Med. 2017;34:851-4.

26. Huckvale K, Adomaviciute S, Prieto JT, et al. Smartphone apps for calculating insulin dose: a systematic assessment. BMC Med. 2015;13:106. https://doi.org/10.1186/s12916-015-0314-7.

27. Davidson MB. Delayed response to NPH insulin. Diabetes Res Clin Pract. 2004;64:229.

28. Davidson MB. Delayed response to NPH insulin. In: Draznin B, editor. Diabetes case files: real problems, practical solutions. Alexandria: American Diabetes Association; 2014. p. 267-70.

29. Davidson MB. Delayed response to U-500 regular insulin. Clin Diabetes. 2018;36:77-9.

30. Harper R, Donnelly R, Bi Y, et al. Dynamics in insulin requirements and treatment safety. J Diabetes Compl. 2016;30:1333-8.
31. Edelman SV, Liu R, Johnson J, Glass LG. AUTONOMY: the first randomized trial comparing two patient-driven approaches to initiate and titrate prandial insulin lispro in type 2 diabetes. Diabetes Care. 2014;37:2132-40.

32. Hodish I. Insulin therapy, weight gain and prognosis. Diabetes Obes Metab. 2018;20:2085-92.

33. Calvert MJ, McManis RJ, Freemantle N. Management of type 2 diabetes with multiple oral hyperglycaemic agents or insulin in primary care retrospective cohort study. $\mathrm{Br} \mathrm{J}$ Gen Pract. 2007;57:455-60.

34. Rubino A, McQuay LJ, Gough SC, et al. Delayed initiation of subcutaneous insulin therapy after failure of oral glucose-lowering agents in patients with type 2 diabetes: a population-based analysis in the UK. Diabet Med. 2007;24:1412-8.

35. Nichols GA, Koo YH, Shah SN. Delay of insulin addition to oral combination therapy despite inadequate glycemic control. J Gen Intern Med. 2007;22:453-8.

36. Khunti K, Wolden MI, Thorsted BL, et al. Clinical inertia in people with type 2 diabetes: a retrospective cohort study of more than 80,000 people. Diabetes Care. 2013;36:3411-7.

37. Home P, Naggar NE, Khamseh $M$, et al. An observational non-interventional study of people with diabetes beginning or changed to insulin analogue therapy in non-Western countries: the A1chieve study. Diabetes Res Clin Pract. 2011;94:352-63.

38. Harris SB, Kapor J, Lank CN, et al. Clinical inertia in patients with T2DM requiring insulin in family practice. Can Fam Physician. 2010;56:e418-24.

39. Yang Y, Lang Q, Jackson SL, et al. Nurse practitioners, physician assistants, and physicians are comparable in managing the first five years of diabetes. Am J Med. 2018;131:276-83.

40. Balkau B, Calvi-Gries F, Freemantle N, Vincent M, Pilorget V, Home PD. Predictors of HbA1c over 4 years in people with type 2 diabetes starting insulin therapies: the CREDIT study. Diabetes Res Clin Pract. 2015;108:432-40.

41. Ziemer DC, Miller CD, Rhee MK, et al. Clinical inertia contributes to poor diabetes control in a primary care setting. Diabetes Educ. 2005;31:564-71.

42. Chen Y, Abbott S, Nguyen M, et al. Glycemic control of insulin treated patients across the U.S.: epidemiologic analysis of a commercially insured population. Diabetes. 2013;62(Suppl 1):A704. 
43. American Diabetes Association. Economic costs of diabetes in the U.S. in 2017. Diabetes Care. 2018;41:917-28.

44. The Diabetes Control and Complications Trial Research Group. The effect of intensive treatment of diabetes on the development and progression of long-term complications in insulin-dependent diabetes mellitus. N Engl J Med. 1993;329:977-86.

45. Gilmer TP, O'Connor PJ, Manning WG, et al. The cost to health plans of poor glycemic control. Diabetes Care. 1997;20:1847-53. 